\title{
Extending Derivations
}

\author{
By \\ C. J. K. BATTY*, A. L. CAREY**, D. E. EvanS***, \\ and Derek W. RoBInson**
}

\section{§1. Introduction}

If $\tau$ is the action of a compact abelian group $G$ on a $C^{*}$-algebra $\mathscr{A}$, and $\delta$ is a derivation on $\mathscr{A}$ commuting with $\tau$, then there has been much interest recently in the problem of deciding when $\delta$ is a generator, under some conditions on the $C^{*}$-dynamical system ( $\mathscr{A}$, $G, \tau)$ and on the restriction of the derivation to the fixed point algebra, see e.g. [1-6, 8-10,12]. Here we consider the problem of deciding when a given derivation on the fixed point algebra extends to a derivation on $\mathscr{A}$ which commutes with the group action. In particular, let $\mathscr{D}$ be a ${ }^{*}$-subalgebra of $\mathscr{A}$, (assumed unital), which contains a unitary $u(\gamma)$ in each spectral subspace $\mathscr{A}^{\tau}(\gamma), \gamma \in \hat{G}$, and such that $\delta_{0}$ is a densely defined derivation on $\mathscr{D} \cap \mathscr{A}^{\tau}$. Suppose that there exists a family of traces on $\mathscr{A}$ which separate its centre. Then we show that $\delta_{0}$ extends to a derivation on $\mathscr{D}$ if and only if both of the following conditions hold:

(1.1) $u(\gamma) \delta_{0}\left(u(\gamma)^{*}(\cdot) u(\gamma)\right) u(\gamma)^{*}-\delta_{0}(\cdot)$ is a bounded inner derivation on $\mathscr{A}$ for all $\gamma$ in $\hat{G}$.

(1. 2) $\varphi\left[\delta_{0}\left(u\left(\gamma_{1}\right) * u\left(\gamma_{2}\right) * u\left(\gamma_{1}\right) u\left(\gamma_{2}\right)\right) u\left(\gamma_{2}\right) * u\left(\gamma_{1}\right) * u\left(\gamma_{2}\right) u\left(\gamma_{1}\right)\right]=0$

for any trace $\varphi$ on $\mathscr{A}, \gamma_{1}, \gamma_{2} \in \hat{G}$.

Our technique is to produce a cohomological obstruction to extending $\delta_{0}$ and to show that this obstruction vanishes in the circumstances of the preceding paragraph. We note further that in

Communicated by H. Araki, December 28, 1982.

* Department of Mathematics, University of Edinburgh, Edinburgh, EH9 3JZ, Scotland.

** Department of Mathematics, Institute of Advanced Studies,

Australian National University, Canberra, ACT 2602, Australia.

*** Mathematics Institute, University of Warwick, Coventry, CV4 7AL, England. 
fact the extension problem for $\delta_{0}$ is equivalent to a problem on group extensions.

\section{§ 2. Preliminaries}

If $G$ is a compact abelian group, an action $\tau$ of $G$ on a $C^{*}$ algebra $\mathscr{A}$ will be a homomorphism $\tau$ from $G$ into Aut $(\mathscr{A})$, the group of all $*$-automorphisms of $\mathscr{A}$, which is strongly continuous in the sense that $g \rightarrow \tau(g)(x)$ is norm continuous for each $x$ in $\mathscr{A}$. If $\gamma \in \Gamma$, the dual group of $G$, the spectral subspace corresponding to $\gamma$ is

$$
\mathscr{A}^{\tau}(\gamma)=\{x \in \mathrm{A}: \tau(g)(x)=\langle\gamma, g\rangle x, g \in G\} \text {. }
$$

We write $\mathscr{A}^{\tau}$ for the fixed point algebra. Then

$$
\mathscr{A}^{\tau}\left(\gamma_{1}\right) \mathscr{A}^{\tau}\left(\gamma_{2}\right) \subseteq \mathscr{A}^{\tau}\left(\gamma_{1}+\gamma_{2}\right), \mathscr{A}^{\tau}(\gamma) *=\mathscr{A}^{\tau}(-\gamma) \text {. }
$$

If $\mathscr{A}$ is a $C^{*}$-algebra, always assumed unital, $\mathscr{Z}(\mathscr{A})$ will denote its centre and $\mathscr{A}_{h}$ its hermitian elements. A derivation on $\mathscr{A}$ will be a linear map $\delta$ defined on a dense ${ }^{*}$-subalgebra $\mathscr{D}$ of $\mathscr{A}$, containing the unit of $\mathscr{A}$, into $\mathscr{A}$ satisfying

$$
\begin{aligned}
& \delta(x y)=\delta(x) y+x \delta(y) \\
& \delta\left(x^{*}\right)=\delta(x)^{*}, \quad x, y \in \mathscr{A} .
\end{aligned}
$$

If $\varphi$ is a trace on $\mathscr{A}$, and $u, v$ unitaries in $\mathscr{D}$, then [13]:

$$
\varphi\left[\delta(u v) v^{*} u^{*}\right]=\varphi\left[\delta(u) u^{*}\right]+\varphi\left[\delta(v) v^{*}\right] .
$$

Moreover

$$
\delta\left(u^{*}\right)=-u^{*} \delta(u) u^{*}
$$

\section{§ 3. Extending Derivations}

Let $(\mathscr{A}, G, \tau)$ be a $C^{*}$-dynamical system where $\tau$ is an action of a compact abelian group $G$ on a unital $C^{*}$-algebra $\mathscr{A}$, and $\delta_{0}$ a derivation on $\mathscr{A}^{\tau}$ with domain $\mathscr{D}_{0}$.

Suppose that there exist unitaries $u(\gamma)$ in $\mathscr{A}^{\tau}(\gamma)$ for each $\gamma$ in $\Gamma$ such that

$$
\begin{gathered}
u(0)=1 \\
u\left(\gamma_{1}\right) u\left(\gamma_{2}\right) u\left(\gamma_{1}+\gamma_{2}\right) * \in \mathscr{D}_{0}, \quad \gamma_{1}, \gamma_{2} \in \Gamma \\
u(\gamma) \mathscr{D}_{0} u(\gamma)^{*} \subseteq \mathscr{D}_{0}, \quad \gamma \in \Gamma .
\end{gathered}
$$


Then

$$
u(\gamma) u(-\gamma) \in \mathscr{D}_{0}
$$

and

$$
\begin{aligned}
u(\gamma) * \mathscr{D}_{0} u(\gamma) & =u(-\gamma)[u(\gamma) u(-\gamma)]^{*} \mathscr{D}_{0}[u(\gamma) u(-\gamma)] u(-\gamma) * \\
& \subseteq \mathscr{D}_{0}
\end{aligned}
$$

and so

$$
u(\gamma) \mathscr{D}_{0} u(\gamma) *=\mathscr{D}_{0}=u(\gamma) * \mathscr{D}_{0} u(\gamma)
$$

Moreover

$$
u(\gamma) *=[u(-\gamma) u(\gamma)] * u(-\gamma)
$$

Thus

$$
\mathscr{D}=\operatorname{lin}\left\{\mathscr{D}_{0} u(\gamma): \gamma \in \hat{G}\right\}
$$

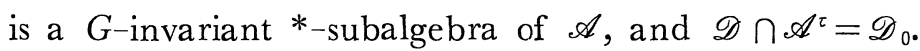

Consider the following condition on the family $\{u(\gamma): \gamma \in \Gamma\}$ :

Hypothesis 3.1. For each $\gamma$ in $\Gamma$, there exists a self adjoint $b(\gamma)$ in $\mathscr{A}^{\tau}$ such that

$$
\begin{gathered}
u(\gamma) \delta_{0}(u(\gamma) * x u(\gamma)) u(\gamma)^{*}-\delta_{0}(x)=i[b(\gamma), x], \quad x \in \mathscr{D}_{0} \\
b(0)=0 .
\end{gathered}
$$

The following shows that this condition is essentially independent of the choice of unitaries in the spectral subspaces. Let $\{v(\gamma): \gamma \in \Gamma\}$ be another family of unitaries in $\mathscr{A}^{\tau}(\gamma)$ with $c(\gamma)=u(\gamma) v(\gamma) * \in \mathscr{D}_{0}$, $c(0)=1$, so that $v$ satisfies $(3.1-3)$. Then $\mathscr{D}=\operatorname{lin}\left\{\mathscr{D}_{0} v(\gamma): \gamma \in \Gamma\right\}$. (Equivalently, for each $\gamma$ in $\Gamma$, let $v(\gamma)$ be a unitary in $\mathscr{D} \cap \mathscr{A}^{\tau}(\gamma)$, with $v(0)=1$ ).

Lemma 3.2. Let $u(\cdot)$ and $v(\cdot)$ be as above. Then Hypothesis 3. 1 holds for $\{u(\gamma): \gamma \in \Gamma\}$ if and only if it holds for $\{v(\gamma): \gamma \in \Gamma\}$.

Proof. For $x \in \mathscr{D}_{0}$ :

$$
\begin{aligned}
u(\gamma) & \delta_{0}[u(\gamma) * x u(\gamma)] u(\gamma) *-\delta_{0}(x) \\
= & c(\gamma) v(\gamma) \delta_{0}\left[v(\gamma) *_{c}(\gamma) *_{v}(\gamma) v(\gamma) * x v(\gamma) v(\gamma) *_{c}(\gamma) v(\gamma)\right] v(\gamma) *_{c}(\gamma) * \\
& -\delta_{0}(x) \\
= & c(\gamma) v(\gamma)\left\{\delta_{0}\left[v(\gamma) *_{c}(\gamma) *_{v}(\gamma)\right] v(\gamma) * x v(\gamma) v(\gamma) *_{c}(\gamma) v(\gamma)\right. \\
& +v(\gamma) *_{c}(\gamma) * v(\gamma) \delta_{0}[v(\gamma) * x v(\gamma)] v(\gamma) * c(\gamma) v(\gamma) \\
& \left.+v(\gamma) *_{c}(\gamma) * v(\gamma) v(\gamma) * x v(\gamma) \delta_{0}\left[v(\gamma) *_{c}(\gamma) v(\gamma)\right]\right\} v(\gamma) *_{c}(\gamma) * \\
& -\delta_{0}(x)
\end{aligned}
$$




$$
=v(\gamma) \delta_{0}[v(\gamma) * x v(\gamma)] v(\gamma) *-\delta_{0}(x)+[d(\gamma), x]
$$

if

$$
\begin{aligned}
d(\gamma) & =c(\gamma) v(\gamma) \delta_{0}[v(\gamma) * c(\gamma) * v(\gamma)] v(\gamma) * \\
& =-v(\gamma) \delta_{0}[v(\gamma) * c(\gamma) v(\gamma)] v(\gamma) * c(\gamma) * \in \mathscr{A}^{\tau},
\end{aligned}
$$

where $d(0)=0$.

Under the conditions of Hypothesis 3.1, define $\Phi=\Phi_{u}: \Gamma^{2} \rightarrow \mathscr{A}^{\tau}$ by

$$
\begin{aligned}
\Phi\left(\gamma_{1}, \gamma_{2}\right)= & b\left(\gamma_{1}+\gamma_{2}\right)-b\left(\gamma_{1}\right)-u\left(\gamma_{1}\right) b\left(\gamma_{2}\right) u\left(\gamma_{1}\right) * \\
& +i u\left(\gamma_{1}+\gamma_{2}\right) \delta_{0}\left[u\left(\gamma_{1}+\gamma_{2}\right) * u\left(\gamma_{1}\right) u\left(\gamma_{2}\right)\right] u\left(\gamma_{2}\right) * u\left(\gamma_{1}\right) *
\end{aligned}
$$

for $\gamma_{1}, \gamma_{2} \in \Gamma$.

Lemma 3. 3. $\Phi\left(\gamma_{1}, \gamma_{2}\right)$ is self adjoint and lies in the centre $\mathscr{Z}\left(\mathscr{A}^{t}\right)$ of $\mathscr{A}^{\tau}$, for all $\gamma_{1}, \gamma_{2}$ in $\Gamma$.

Proof. That $\Phi\left(\gamma_{1}, \gamma_{2}\right)$ is self adjoint follows easily from (2. 2). Then for $x \in \mathscr{D}_{0}$, using (3.5):

$$
\begin{aligned}
i\left[\Phi\left(\gamma_{1}, \gamma_{2}\right), x\right]= & u\left(\gamma_{1}+\gamma_{2}\right) \delta_{0}\left(u\left(\gamma_{1}+\gamma_{2}\right) * x u\left(\gamma_{1}+\gamma_{2}\right)\right) u\left(\gamma_{1}+\gamma_{2}\right) *-\delta_{0}(x) \\
& -u\left(\gamma_{1}\right) \delta_{0}\left(u\left(\gamma_{1}\right) * x u\left(\gamma_{1}\right)\right) u\left(\gamma_{1}\right) * \delta_{0}(x) \\
& -u\left(\gamma_{1}\right) u\left(\gamma_{2}\right) \delta_{0}\left(u\left(\gamma_{2}\right) * u\left(\gamma_{1}\right) * x u\left(\gamma_{1}\right) u\left(\gamma_{2}\right)\right) u\left(\gamma_{2}\right) * u\left(\gamma_{1}\right) * \\
& +u\left(\gamma_{1}\right) \delta_{0}\left(u\left(\gamma_{1}\right) * x u\left(\gamma_{1}\right)\right) u\left(\gamma_{1}\right) * \\
& -u\left(\gamma_{1}+\gamma_{2}\right) \delta_{0}\left(u\left(\gamma_{1}+\gamma_{2}\right) * u\left(\gamma_{1}\right) u\left(\gamma_{2}\right)\right) u\left(\gamma_{2}\right) * u\left(\gamma_{1}\right) * x \\
& +x u\left(\gamma_{1}+\gamma_{2}\right) \delta_{0}\left(u\left(\gamma_{1}+\gamma_{2}\right) * u\left(\gamma_{1}\right) u\left(\gamma_{2}\right)\right) u\left(\gamma_{2}\right) * u\left(\gamma_{1}\right) * \\
= & u\left(\gamma_{1}+\gamma_{2}\right) \delta_{0}\left\{\left[u\left(\gamma_{1}+\gamma_{2}\right) * u\left(\gamma_{1}\right) u\left(\gamma_{2}\right)\right]\right. \\
& \cdot\left[u\left(\gamma_{2}\right) * u\left(\gamma_{1}\right) * x u\left(\gamma_{1}\right) u\left(\gamma_{2}\right)\right] \\
& \left.\cdot\left[u\left(\gamma_{2}\right) * u\left(\gamma_{1}\right) * u\left(\gamma_{1}+\gamma_{2}\right)\right]\right\} u\left(\gamma_{1}+\gamma_{2}\right) * \\
& -u\left(\gamma_{1}\right) u\left(\gamma_{2}\right) \delta_{0}\left[u\left(\gamma_{2}\right) * u\left(\gamma_{1}\right) * x u\left(\gamma_{1}\right) u\left(\gamma_{2}\right)\right] u\left(\gamma_{2}\right) * u\left(\gamma_{1}\right) * \\
& -u\left(\gamma_{1}+\gamma_{2}\right) \delta_{0}\left[u\left(\gamma_{1}+\gamma_{2}\right) * u\left(\gamma_{1}\right) u\left(\gamma_{2}\right)\right] u\left(\gamma_{2}\right) * u\left(\gamma_{1}\right) * x \\
& +x u\left(\gamma_{1}+\gamma_{2}\right) \delta_{0}\left[u\left(\gamma_{1}+\gamma_{2}\right) * u\left(\gamma_{1}\right) u\left(\gamma_{2}\right)\right] u\left(\gamma_{2}\right) * u\left(\gamma_{1}\right) * \\
= & 0
\end{aligned}
$$

Lemma 3.4. $\Phi$ is a u-twisted 2-cocycle, i.e., (3. 8) $\Phi\left(\gamma_{1}+\gamma_{2}, \gamma_{3}\right)+\Phi\left(\gamma_{1}, \gamma_{2}\right)=\Phi\left(\gamma_{1}, \gamma_{2}+\gamma_{3}\right)+u\left(\gamma_{1}\right) \Phi\left(\gamma_{2}, \gamma_{3}\right) u\left(\gamma_{1}\right) *$ for all $\gamma_{1}, \gamma_{2}, \gamma_{3}$ in $\Gamma$.

\section{Proof.}

$\Phi\left(\gamma_{1}+\gamma_{2}, \gamma_{3}\right)+\Phi\left(\gamma_{1}, \gamma_{2}\right)-\Phi\left(\gamma_{1}, \gamma_{2}+\gamma_{3}\right)-u\left(\gamma_{1}\right) \Phi\left(\gamma_{2}, \gamma_{3}\right) u\left(\gamma_{1}\right)^{*}$ 


$$
\begin{aligned}
& =b\left(\gamma_{1}+\gamma_{2}+\gamma_{3}\right)-b\left(\gamma_{1}+\gamma_{2}\right)-u\left(\gamma_{1}+\gamma_{2}\right) b\left(\gamma_{3}\right) u\left(\gamma_{1}+\gamma_{2}\right) * \\
& +i u\left(\gamma_{1}+\gamma_{2}+\gamma_{3}\right) \delta_{0}\left[u\left(\gamma_{1}+\gamma_{2}+\gamma_{3}\right) * u\left(\gamma_{1}+\gamma_{2}\right) u\left(\gamma_{3}\right)\right] u\left(\gamma_{3}\right) * u\left(\gamma_{1}+\gamma_{2}\right) * \\
& +b\left(\gamma_{1}+\gamma_{2}\right)-b\left(\gamma_{1}\right)-u\left(\gamma_{1}\right) b\left(\gamma_{2}\right) u\left(\gamma_{1}\right)^{*} \\
& +i u\left(\gamma_{1}+\gamma_{2}\right) \delta_{0}\left[u\left(\gamma_{1}+\gamma_{2}\right) * u\left(\gamma_{1}\right) u\left(\gamma_{2}\right)\right] u\left(\gamma_{2}\right) * u\left(\gamma_{1}\right) * \\
& -b\left(\gamma_{1}+\gamma_{2}+\gamma_{3}\right)+b\left(\gamma_{1}\right)+u\left(\gamma_{1}\right) b\left(\gamma_{2}+\gamma_{3}\right) u\left(\gamma_{1}\right) * \\
& -i u\left(\gamma_{1}+\gamma_{2}+\gamma_{3}\right) \delta_{0}\left[u\left(\gamma_{1}+\gamma_{2}+\gamma_{3}\right) * u\left(\gamma_{1}\right) u\left(\gamma_{2}+\gamma_{3}\right)\right] u\left(\gamma_{2}+\gamma_{3}\right) * u\left(\gamma_{1}\right) * \\
& -u\left(\gamma_{1}\right) b\left(\gamma_{2}+\gamma_{3}\right) u\left(\gamma_{1}\right)^{*}+u\left(\gamma_{1}\right) b\left(\gamma_{2}\right) u\left(\gamma_{1}\right)^{*} \\
& +u\left(\gamma_{1}\right) u\left(\gamma_{2}\right) b\left(\gamma_{3}\right) u\left(\gamma_{2}\right) * u\left(\gamma_{1}\right) * \\
& -i u\left(\gamma_{1}\right) u\left(\gamma_{2}+\gamma_{3}\right) \delta_{0}\left[u\left(\gamma_{2}+\gamma_{3}\right) * u\left(\gamma_{2}\right) u\left(\gamma_{3}\right)\right] u\left(\gamma_{3}\right) * u\left(\gamma_{2}\right) * u\left(\gamma_{1}\right) * \\
& =u\left(\gamma_{1}\right) u\left(\gamma_{2}\right)\left[b\left(\gamma_{3}\right), u\left(\gamma_{2}\right) * u\left(\gamma_{1}\right) * u\left(\gamma_{1}+\gamma_{2}\right)\right] u\left(\gamma_{1}+\gamma_{2}\right) * \\
& +i u\left(\gamma_{1}+\gamma_{2}+\gamma_{3}\right) \delta_{0}\left[u\left(\gamma_{1}+\gamma_{2}+\gamma_{3}\right) * u\left(\gamma_{1}+\gamma_{2}\right) u\left(\gamma_{3}\right)\right] u\left(\gamma_{3}\right) * u\left(\gamma_{1}+\gamma_{2}\right) * \\
& +i u\left(\gamma_{1}+\gamma_{2}\right) \delta_{0}\left[u\left(\gamma_{1}+\gamma_{2}\right) * u\left(\gamma_{1}\right) u\left(\gamma_{2}\right)\right] u\left(\gamma_{2}\right) * u\left(\gamma_{1}\right) * \\
& -i u\left(\gamma_{1}+\gamma_{2}+\gamma_{3}\right) \delta_{0}\left[u\left(\gamma_{1}+\gamma_{2}+\gamma_{3}\right) * u\left(\gamma_{1}\right) u\left(\gamma_{2}+\gamma_{3}\right)\right] u\left(\gamma_{2}+\gamma_{3}\right) * u\left(\gamma_{1}\right) * \\
& -i u\left(\gamma_{1}\right) u\left(\gamma_{2}+\gamma_{3}\right) \delta_{0}\left[u\left(\gamma_{2}+\gamma_{3}\right) * u\left(\gamma_{2}\right) u\left(\gamma_{3}\right)\right] u\left(\gamma_{3}\right) * u\left(\gamma_{2}\right) * u\left(\gamma_{1}\right) * \\
& =i u\left(\gamma_{1}\right) u\left(\gamma_{2}\right) \delta_{0}\left[u\left(\gamma_{2}\right) * u\left(\gamma_{1}\right) * u\left(\gamma_{1}+\gamma_{2}\right)\right] u\left(\gamma_{1}+\gamma_{2}\right) * \\
& -i u\left(\gamma_{1}\right) u\left(\gamma_{2}\right) u\left(\gamma_{3}\right) \delta_{0}\left[u\left(\gamma_{3}\right) * u\left(\gamma_{2}\right) * u\left(\gamma_{1}\right) * u\left(\gamma_{1}+\gamma_{2}\right) u\left(\gamma_{3}\right)\right] \\
& \text { - } u\left(\gamma_{3}\right) * u\left(\gamma_{1}+\gamma_{2}\right) * \\
& +i u\left(\gamma_{1}+\gamma_{2}+\gamma_{3}\right) \delta_{0}\left[u\left(\gamma_{1}+\gamma_{2}+\gamma_{3}\right) * u\left(\gamma_{1}+\gamma_{2}\right) u\left(\gamma_{3}\right)\right] u\left(\gamma_{3}\right) * u\left(\gamma_{1}+\gamma_{2}\right) * \\
& +i u\left(\gamma_{1}+\gamma_{2}\right) \delta_{0}\left[u\left(\gamma_{1}+\gamma_{2}\right) * u\left(\gamma_{1}\right) u\left(\gamma_{2}\right)\right] u\left(\gamma_{2}\right) * u\left(\gamma_{1}\right) * \\
& -i u\left(\gamma_{1}+\gamma_{2}+\gamma_{3}\right) \delta_{0}\left[u\left(\gamma_{1}+\gamma_{2}+\gamma_{3}\right) * u\left(\gamma_{1}\right) u\left(\gamma_{2}+\gamma_{3}\right)\right] u\left(\gamma_{2}+\gamma_{3}\right) * u\left(\gamma_{1}\right) * \\
& -i u\left(\gamma_{1}\right) u\left(\gamma_{2}+\gamma_{3}\right) \delta_{0}\left[u\left(\gamma_{2}+\gamma_{3}\right) * u\left(\gamma_{2}\right) u\left(\gamma_{3}\right)\right] u\left(\gamma_{3}\right) * u\left(\gamma_{2}\right) * u\left(\gamma_{1}\right) * \\
& =-i u\left(\gamma_{1}\right) u\left(\gamma_{2}\right) u\left(\gamma_{3}\right) \delta_{0}\left[u\left(\gamma_{3}\right) * u\left(\gamma_{2}\right) * u\left(\gamma_{1}\right) * u\left(\gamma_{1}+\gamma_{2}\right) u\left(\gamma_{3}\right)\right] u\left(\gamma_{3}\right) * u\left(\gamma_{1}+\gamma_{2}\right) * \\
& +i u\left(\gamma_{1}+\gamma_{2}+\gamma_{3}\right) \delta_{0}\left[u\left(\gamma_{1}+\gamma_{2}+\gamma_{3}\right) * u\left(\gamma_{1}+\gamma_{2}\right) u\left(\gamma_{3}\right)\right] u\left(\gamma_{3}\right) * u\left(\gamma_{1}+\gamma_{2}\right) * \\
& -i u\left(\gamma_{1}+\gamma_{2}+\gamma_{3}\right) \delta_{0}\left\{\left[u\left(\gamma_{1}+\gamma_{2}+\gamma_{3}\right) * u\left(\gamma_{1}\right) u\left(\gamma_{2}+\gamma_{3}\right)\right]\right. \\
& \text { - } \left.\left[u\left(\gamma_{2}+\gamma_{3}\right) * u\left(\gamma_{2}\right) u\left(\gamma_{3}\right)\right]\right\} u\left(\gamma_{3}\right) * u\left(\gamma_{2}\right) * u\left(\gamma_{1}\right) * \\
& =i u\left(\gamma_{1}+\gamma_{2}+\gamma_{3}\right) \delta_{0}\left[u\left(\gamma_{1}+\gamma_{2}+\gamma_{3}\right) * u\left(\gamma_{1}+\gamma_{2}\right) u\left(\gamma_{3}\right)\right] u\left(\gamma_{3}\right) * u\left(\gamma_{1}+\gamma_{2}\right) * \\
& -i u\left(\gamma_{1}+\gamma_{2}+\gamma_{3}\right) \delta_{0}\left\{\left[u\left(\gamma_{1}+\gamma_{2}+\gamma_{3}\right) * u\left(\gamma_{1}\right) u\left(\gamma_{2}\right) u\left(\gamma_{3}\right)\right]\right. \\
& \text { - } \left.\left[u\left(\gamma_{3}\right) * u\left(\gamma_{2}\right) * u\left(\gamma_{1}\right) * u\left(\gamma_{1}+\gamma_{2}\right) u\left(\gamma_{3}\right)\right]\right\} u\left(\gamma_{3}\right) * u\left(\gamma_{1}+\gamma_{2}\right) * \\
& =0 \text {. }
\end{aligned}
$$

\section{Lemma 3.5. Suppose}

(3.9) $\Phi\left(\gamma_{1}, \gamma_{2}\right)=z\left(\gamma_{1}+\gamma_{2}\right)-z\left(\gamma_{1}\right)-u\left(\gamma_{1}\right) z\left(\gamma_{2}\right) u\left(\gamma_{1}\right) *, \quad \gamma_{1}, \gamma_{2} \in \Gamma$

for some self adjoint family $\{z(\gamma): \gamma \in \Gamma\}$ in $\mathscr{Z}\left(\mathscr{A}^{\tau}\right)$, with $z(0)=0$. Let $b_{0}(\gamma)=b(\gamma)-z(\gamma), \gamma \in \Gamma$. Then

$$
\delta(x u(\gamma))=\delta_{0}(x) u(\gamma)-i x b_{0}(\gamma) u(\gamma), \gamma \in \Gamma, x \in \mathscr{D}_{0}
$$

defines a derivation $\delta$ on $\mathscr{D}$ which commutes with $G$, and extends $\delta_{0}$. 
Proof. The map $b_{0}: \Gamma \rightarrow \mathscr{A}_{h}^{\tau}$ satisfies

(3.11) $u(\gamma) \delta_{0}\left(u(\gamma)^{*} x u(\gamma)\right) u(\gamma)^{*}-\delta_{0}(x)=i\left[b_{0}(\gamma), x\right], x \in \mathscr{D}_{0}$

(3.12) $b_{0}\left(\gamma_{1}+\gamma_{2}\right)=b_{0}\left(\gamma_{1}\right)+u\left(\gamma_{1}\right) b_{0}\left(\gamma_{2}\right) u\left(\gamma_{1}\right) *$

$$
-i u\left(\gamma_{1}+\gamma_{2}\right) \delta_{0}\left[u\left(\gamma_{1}+\gamma_{2}\right) * u\left(\gamma_{1}\right) u\left(\gamma_{2}\right)\right] u\left(\gamma_{2}\right) * u\left(\gamma_{1}\right) *
$$

$$
b_{0}(0)=0 \text {. }
$$

Define $\delta$ by (3.10). Now by (3.12):

$$
0=b_{0}(\gamma)+u(\gamma) b_{0}(-\gamma) u(\gamma) *-i \delta_{0}(u(\gamma) u(-\gamma)) u(-\gamma) * u(\gamma) * .
$$

Then for $x \in \mathscr{D}_{0}, r \in \Gamma$ :

$$
\begin{aligned}
\delta[x u(\gamma)]^{*}= & \delta\left[\left(u(\gamma) * x^{*} u(-\gamma) *\right) u(-\gamma)\right] \\
= & \delta_{0}\left[u(\gamma) * x^{*} u(-\gamma) *\right] u(-\gamma)-i u(\gamma) * x^{*} u(-\gamma) * b_{0}(-\gamma) u(-\gamma) \\
= & \delta_{0}\left(u(\gamma) * x^{*} u(-\gamma) *\right) u(-\gamma) \\
& -i u(\gamma) * x^{*} u(-\gamma) *\left[-u(\gamma) * b_{0}(\gamma) u(\gamma)\right. \\
& \left.+i u(\gamma) * \delta_{0}(u(\gamma) u(-\gamma)) u(-\gamma) *\right] u(-\gamma) \\
= & u(\gamma) * \delta_{0}\left(x^{*} u(-\gamma) * u(\gamma) *\right) u(\gamma) u(-\gamma) \\
& +i u(\gamma) * b_{0}(\gamma) x^{*}-i u(\gamma) * x^{*} u(-\gamma) * u(\gamma) * b_{0}(\gamma) u(\gamma) u(-\gamma) \\
& +i u(\gamma) * x^{*} u(-\gamma) * u(\gamma) * b_{0}(\gamma) u(\gamma) u(-\gamma) \\
& +u(\gamma) * x^{*} u(-\gamma) * u(\gamma) * \delta_{0}(u(\gamma) u(-\gamma)) \\
= & u(\gamma) * \delta_{0}\left(x^{*}\right)+i u(\gamma) * b_{0}(\gamma) x^{*} \\
= & {[\delta(x u(\gamma))]^{*} . }
\end{aligned}
$$

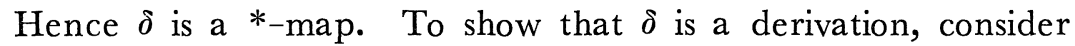
$x_{1}, x_{2} \in \mathscr{D}_{0}, \gamma_{1}, \gamma_{2} \in \Gamma:$

$$
\begin{aligned}
\delta\left(x_{1} u\left(\gamma_{1}\right) x_{2} u\right. & \left.\left(\gamma_{2}\right)\right)-\delta\left(x_{1} u\left(\gamma_{1}\right)\right) x_{2} u\left(\gamma_{2}\right)-x_{1} u\left(\gamma_{1}\right) \delta\left(x_{2} u\left(\gamma_{2}\right)\right) \\
= & \delta\left(x_{1} u\left(\gamma_{1}\right) x_{2} u\left(\gamma_{2}\right) u\left(\gamma_{1}+\gamma_{2}\right) * u\left(\gamma_{1}+\gamma_{2}\right)\right)-\delta\left(x_{1} u\left(\gamma_{1}\right)\right) x_{2} u\left(\gamma_{2}\right) \\
& -x_{1} u\left(\gamma_{1}\right) \delta\left(x_{2} u\left(\gamma_{2}\right)\right) \\
= & \delta_{0}\left[x_{1} u\left(\gamma_{1}\right) x_{2} u\left(\gamma_{2}\right) u\left(\gamma_{1}+\gamma_{2}\right) *\right] u\left(\gamma_{1}+\gamma_{2}\right) \\
& -i x_{1} u\left(\gamma_{1}\right) x_{2} u\left(\gamma_{2}\right) u\left(\gamma_{1}+\gamma_{2}\right) * b_{0}\left(\gamma_{1}+\gamma_{2}\right) u\left(\gamma_{1}+\gamma_{2}\right) \\
& -\delta_{0}\left(x_{1}\right) u\left(\gamma_{1}\right) x_{2} u\left(\gamma_{2}\right)+i x_{1} b_{0}\left(\gamma_{1}\right) u\left(\gamma_{1}\right) x_{2} u\left(\gamma_{2}\right) \\
& -x_{1} u\left(\gamma_{1}\right) \delta_{0}\left(x_{2}\right) u\left(\gamma_{2}\right)+i x_{1} u\left(\gamma_{1}\right) x_{2} b_{0}\left(\gamma_{2}\right) u\left(\gamma_{2}\right) \\
= & x_{1}\left\{\delta_{0}\left[u\left(\gamma_{1}\right) x_{2} u\left(\gamma_{2}\right) u\left(\gamma_{1}+\gamma_{2}\right) *\right] u\left(\gamma_{1}+\gamma_{2}\right)\right. \\
& -i u\left(\gamma_{1}\right) x_{2} u\left(\gamma_{2}\right) u\left(\gamma_{1}+\gamma_{2}\right) * b_{0}\left(\gamma_{1}\right) u\left(\gamma_{1}+\gamma_{2}\right) \\
& -i u\left(\gamma_{1}\right) x_{2} u\left(\gamma_{2}\right) u\left(\gamma_{1}+\gamma_{2}\right) * u\left(\gamma_{1}\right) b_{0}\left(\gamma_{2}\right) u\left(\gamma_{1}\right) * u\left(\gamma_{1}+\gamma_{2}\right) \\
& -u\left(\gamma_{1}\right) x_{2} u\left(\gamma_{2}\right) \delta_{0}\left(u\left(\gamma_{1}+\gamma_{2}\right) * u\left(\gamma_{1}\right) u\left(\gamma_{2}\right)\right) u\left(\gamma_{1}\right) * u\left(\gamma_{2}\right) * u\left(\gamma_{1}+\gamma_{2}\right) \\
& +i b_{0}\left(\gamma_{1}\right) u\left(\gamma_{1}\right) x_{2} u\left(\gamma_{2}\right)-u\left(\gamma_{1}\right) \delta_{0}\left(x_{2}\right) u\left(\gamma_{2}\right) \\
& \left.+i u\left(\gamma_{1}\right) x_{2} b_{0}\left(\gamma_{2}\right) u\left(\gamma_{2}\right)\right\} \\
= & x_{1}\left\{\delta_{0}\left[u\left(\gamma_{1}\right) x_{2} u\left(\gamma_{2}\right) u\left(\gamma_{1}+\gamma_{2}\right) *\right] u\left(\gamma_{1}+\gamma_{2}\right)\right.
\end{aligned}
$$




$$
\begin{aligned}
& +i\left[b_{0}\left(\gamma_{1}\right), u\left(\gamma_{1}\right) x_{2} u\left(\gamma_{2}\right) u\left(\gamma_{1}+\gamma_{2}\right) *\right] u\left(\gamma_{1}+\gamma_{2}\right) \\
& +i u\left(\gamma_{1}\right) x_{2}\left[b_{0}\left(\gamma_{2}\right), u\left(\gamma_{2}\right) u\left(\gamma_{1}+\gamma_{2}\right) * u\left(\gamma_{1}\right)\right] u\left(\gamma_{1}\right) * u\left(\gamma_{1}+\gamma_{2}\right) \\
& -u\left(\gamma_{1}\right) x_{2} u\left(\gamma_{2}\right) \delta_{0}\left[u\left(\gamma_{1}+\gamma_{2}\right) * u\left(\gamma_{1}\right) u\left(\gamma_{2}\right)\right] u\left(\gamma_{1}\right) * u\left(\gamma_{2}\right) * u\left(\gamma_{1}+\gamma_{2}\right) \\
& \left.-u\left(\gamma_{1}\right) \delta_{0}\left(x_{2}\right) u\left(\gamma_{2}\right)\right\} \\
= & x_{1}\left\{\delta_{0}\left[u\left(\gamma_{1}\right) x_{2} u\left(\gamma_{2}\right) u\left(\gamma_{1}+\gamma_{2}\right) *\right] u\left(\gamma_{1}+\gamma_{2}\right)\right. \\
& +u\left(\gamma_{1}\right) \delta_{0}\left[x_{2} u\left(\gamma_{2}\right) u\left(\gamma_{1}+\gamma_{2}\right) * u\left(\gamma_{1}\right)\right] u\left(\gamma_{1}\right) * u\left(\gamma_{1}+\gamma_{2}\right) \\
& -\delta_{0}\left[u\left(\gamma_{1}\right) x_{2} u\left(\gamma_{2}\right) u\left(\gamma_{1}+\gamma_{2}\right) *\right] u\left(\gamma_{1}+\gamma_{2}\right) \\
& +u\left(\gamma_{1}\right) x_{2} u\left(\gamma_{2}\right) \delta_{0}\left[u\left(\gamma_{1}+\gamma_{2}\right) * u\left(\gamma_{1}\right) u\left(\gamma_{2}\right)\right] u\left(\gamma_{2}\right) * u\left(\gamma_{1}\right) * u\left(\gamma_{1}+\gamma_{2}\right) \\
& -u\left(\gamma_{1}\right) x_{2} \delta_{0}\left[u\left(\gamma_{2}\right) u\left(\gamma_{1}+\gamma_{2}\right) * u\left(\gamma_{1}\right)\right] u\left(\gamma_{1}\right) * u\left(\gamma_{1}+\gamma_{2}\right) \\
& -u\left(\gamma_{1}\right) x_{2} u\left(\gamma_{2}\right) \delta_{0}\left[u\left(\gamma_{1}+\gamma_{2}\right) * u\left(\gamma_{1}\right) u\left(\gamma_{2}\right)\right] u\left(\gamma_{1}\right) * u\left(\gamma_{2}\right) * u\left(\gamma_{1}+\gamma_{2}\right) \\
& \left.-u\left(\gamma_{1}\right) \delta_{0}\left(x_{2}\right) u\left(\gamma_{2}\right)\right\} \\
= & 0 .
\end{aligned}
$$

The following lemma shows that at least all symmetric $\Phi$ which we have considered can be decomposed.

Lemma 3.6. Let $D$ be a divisible abelian group and $\Phi: \Gamma \times \Gamma \rightarrow D$ a symmetric 2-cocycle, i.e.,

$$
\Phi\left(\gamma_{1}+\gamma_{2}, \gamma_{3}\right)+\Phi\left(\gamma_{1}, \gamma_{2}\right)=\Phi\left(\gamma_{1}, \gamma_{2}+\gamma_{3}\right)+\Phi\left(\gamma_{2}, \gamma_{3}\right), \gamma_{i} \in \Gamma
$$

$$
\begin{aligned}
& \Phi\left(\gamma_{1}, 0\right)=0=\Phi\left(0, \gamma_{1}\right), \quad \gamma_{1} \in \Gamma . \\
& \Phi\left(\gamma_{1}, \gamma_{2}\right)=\Phi\left(\gamma_{2}, \gamma_{1}\right), \quad \gamma_{1}, \gamma_{2} \in \Gamma .
\end{aligned}
$$

Then there exists $z: \Gamma \rightarrow D$ such that

$$
\begin{gathered}
\Phi\left(\gamma_{1}, \gamma_{2}\right)=z\left(\gamma_{1}+\gamma_{2}\right)-z\left(\gamma_{1}\right)-z\left(\gamma_{2}\right), \quad \gamma_{1}, \gamma_{2} \in \Gamma . \\
z(0)=0 .
\end{gathered}
$$

Proof. Define a new group $\tilde{\Gamma}=\{(\gamma, b): \gamma \in \Gamma, b \in D\}$ with addition

$$
\left(\gamma_{1}, b_{1}\right)+\left(\gamma_{2}, b_{2}\right)=\left(\gamma_{1}+\gamma_{2}, b_{1}+b_{2}+\Phi\left(\gamma_{1}, \gamma_{2}\right)\right) \text {. }
$$

and inverse: $-\left(\gamma_{1}, b_{1}\right)=\left(-\gamma_{1},-b_{1}-\Phi\left(\gamma_{1},-\gamma_{1}\right)\right)$. Then $\tilde{\Gamma}$ is abelian because $\Phi$ is symmetric.

Now [7] if $K$ is a subgroup of a (discrete) abelian group $H$, then any homomorphism of $K$ into a divisible abelian group $D$, extends to $H$. Taking $H=\tilde{\Gamma}, K=D$, this means that there exists a homomorphism $\eta: \widetilde{\Gamma} \rightarrow D$ extending the identity map from $D$ into $D$. Taking $z(\gamma)=-\eta(\gamma, 0)$, we have the result. 
Remark 3.7. In order to measure the obstruction to $\Phi$ (defined by (3.7)) being a coboundary, it will be useful to look at

$$
\varphi\left\{\delta_{0}\left[u\left(\gamma_{1}\right) * u\left(\gamma_{2}\right) * u\left(\gamma_{1}\right) u\left(\gamma_{2}\right)\right] u\left(\gamma_{2}\right) * u\left(\gamma_{1}\right) * u\left(\gamma_{2}\right) u\left(\gamma_{1}\right)\right\}
$$

if $\varphi$ is a trace on $\mathscr{A}$. It is useful to note that this expression does not depend on the choice of family $\{u(\gamma): \gamma \in \Gamma\}$. Thus let $\{u(\gamma)$ : $\gamma \in \Gamma\}$ be a family of unitaries satisfying (3.1-3), such that Hypothesis 3.1 holds. Let $v(\gamma)$ be another family of unitaries in $\mathscr{D} \cap \mathscr{A}^{\tau}(\gamma)$. Then if $d(\gamma)=v(\gamma) * u(\gamma) \in \mathscr{D}_{0}:$

$$
\begin{aligned}
& u\left(\gamma_{1}\right) * u\left(\gamma_{2}\right) * u\left(\gamma_{1}\right) u\left(\gamma_{2}\right) \\
&=d\left(\gamma_{1}\right) *\left[v\left(\gamma_{1}\right) * d\left(\gamma_{2}\right) * v\left(\gamma_{1}\right)\right]\left[v\left(\gamma_{1}\right) * v\left(\gamma_{2}\right) * v\left(\gamma_{1}\right) v\left(\gamma_{2}\right)\right] \\
& \cdot\left[v\left(\gamma_{2}\right) * d\left(\gamma_{1}\right) v\left(\gamma_{2}\right)\right] d\left(\gamma_{2}\right) .
\end{aligned}
$$

Hence by (2. 1),

$$
\begin{aligned}
& \varphi\left[\delta_{0}\left(u\left(\gamma_{1}\right) * u\left(\gamma_{2}\right) * u\left(\gamma_{1}\right) u\left(\gamma_{2}\right)\right) u\left(\gamma_{2}\right) * u\left(\gamma_{1}\right) * u\left(\gamma_{2}\right) u\left(\gamma_{1}\right)\right] \\
&=\left.\varphi\left[\delta_{0}\left(d\left(\gamma_{1}\right) *\right) d\left(\gamma_{1}\right)\right]+\varphi\left[\delta_{0}\left(v\left(\gamma_{1}\right) * d\left(\gamma_{2}\right) * v\left(\gamma_{1}\right)\right) v\left(\gamma_{1}\right) * d\left(\gamma_{2}\right) v\left(\gamma_{1}\right)\right)\right] \\
&+\varphi\left[\delta_{0}\left(v\left(\gamma_{1}\right) * v\left(\gamma_{2}\right) * v\left(\gamma_{1}\right) v\left(\gamma_{2}\right)\right) v\left(\gamma_{2}\right) * v\left(\gamma_{1}\right) * v\left(\gamma_{2}\right) v\left(\gamma_{1}\right)\right] \\
&+\varphi\left[\delta_{0}\left(v\left(\gamma_{2}\right) * d\left(\gamma_{1}\right) v\left(\gamma_{2}\right)\right) v\left(\gamma_{2}\right) * d\left(\gamma_{1}\right) * v\left(\gamma_{2}\right)\right] \\
&+\varphi\left[\delta_{0}\left(d\left(\gamma_{2}\right)\right) d\left(\gamma_{2}\right) *\right] \\
&= \varphi\left[\delta_{0}\left(d\left(\gamma_{1}\right) *\right) d\left(\gamma_{1}\right)\right]+\varphi\left[v\left(\gamma_{1}\right) * \delta_{0}\left(d\left(\gamma_{2}\right) *\right) v\left(\gamma_{1}\right) v\left(\gamma_{1}\right) * d\left(\gamma_{2}\right) v\left(\gamma_{1}\right)\right] \\
&+\varphi\left\{v\left(\gamma_{1}\right) * i\left[e\left(\gamma_{1}\right), d\left(\gamma_{2}\right) *\right] v\left(\gamma_{1}\right) v\left(\gamma_{1}\right) * d\left(\gamma_{2}\right) v\left(\gamma_{1}\right)\right\} \\
&+\varphi\left[\delta_{0}\left(v\left(\gamma_{1}\right) * v\left(\gamma_{2}\right) * v\left(\gamma_{1}\right) v\left(\gamma_{2}\right)\right) v\left(\gamma_{2}\right) * v\left(\gamma_{1}\right) * v\left(\gamma_{2}\right) v\left(\gamma_{1}\right)\right] \\
&+\varphi\left[v\left(\gamma_{2}\right) * \delta_{0}\left(d\left(\gamma_{1}\right)\right) v\left(\gamma_{2}\right) v\left(\gamma_{2}\right) * d\left(\gamma_{1}\right) * v\left(\gamma_{2}\right)\right] \\
&+\varphi\left[v\left(\gamma_{2}\right) * i\left[e\left(\gamma_{2}\right), d\left(\gamma_{1}\right)\right] v\left(\gamma_{2}\right) v\left(\gamma_{2}\right) * d\left(\gamma_{1}\right) * v\left(\gamma_{2}\right)\right] \\
&+\varphi\left[\delta_{0}\left(d\left(\gamma_{2}\right)\right) d\left(\gamma_{2}\right) *\right] \\
&= \varphi\left[\delta_{0}\left(v\left(\gamma_{1}\right) * v\left(\gamma_{2}\right) * v\left(\gamma_{1}\right) v\left(\gamma_{2}\right)\right) v\left(\gamma_{2}\right) * v\left(\gamma_{1}\right) * v\left(\gamma_{2}\right) v\left(\gamma_{1}\right)\right]
\end{aligned}
$$

where we have used

$$
\delta_{0}(v(\gamma) * x v(\gamma))-v(\gamma) * \delta_{0}(x) v(\gamma)=v(\gamma) * i[e(\gamma), x] v(\gamma)
$$

for $x \in \mathscr{D}_{0}$ and some family $e(\gamma)$ in $\mathscr{A}_{h}^{\tau}$.

Theorem 3.8. Let $\tau$ be an action of a compact abelian group on a $C^{*}$-algebra $\mathscr{A}, \delta_{0}$ be a derivation on $\mathscr{A}^{\tau}$ with domain $\mathscr{D}_{0}$, and suppose that there exists a family $\{u(\gamma): \gamma \in \Gamma\}$ of unitaries in $\mathscr{A}^{\tau}(\gamma)$ satisfying (3.1-3). Suppose that

(3.20) there exists a family of traces on $\mathscr{A}$, which separates $\mathscr{Z}\left(\mathscr{A}^{\tau}\right)$. 
Then $\delta_{0}$ extends to a derivation on

$$
\mathscr{D}=\operatorname{lin}\left\{\mathscr{D}_{0} u(\gamma): \gamma \in \Gamma\right\}
$$

which commutes with $G$, if and only if both the following hold:

(3.21) There exists a family $\{b(\gamma): \gamma \in \Gamma\}$ of self adjoint elements of $\mathscr{A}^{\tau}$ such that

$$
u(\gamma) \delta_{0}(u(\gamma) * x u(\gamma)) u(\gamma) *-\delta_{0}(x)=i[b(\gamma), x]
$$

for all $x$ in $\mathscr{D}_{0}, \gamma \in \Gamma$.

(3. 22) $\varphi\left[\delta_{0}\left(u\left(\gamma_{1}\right) * u\left(\gamma_{2}\right) * u\left(\gamma_{1}\right) u\left(\gamma_{2}\right)\right) u\left(\gamma_{2}\right) * u\left(\gamma_{1}\right) * u\left(\gamma_{2}\right) u\left(\gamma_{1}\right)\right]=0$

for any trace $\varphi$ on $\mathscr{A}, \gamma_{1}, \gamma_{2} \in \Gamma$.

If $\delta_{0}$ is closable, then so is $\delta$.

Proof. We claim that $\mathscr{Z}\left(\mathscr{A}^{\tau}\right) \subseteq \mathscr{Z}(\mathscr{A})$ (cf. [2]). Let $u$ be any unitary in $\mathscr{A}^{\tau}(\gamma)$ for some $\gamma$ in $\Gamma$. Then $u z u^{*} \in \mathscr{Z}\left(\mathscr{A}^{\tau}\right)$ for any $z \in \mathscr{Z}\left(\mathscr{A}^{\tau}\right)$. If $\varphi$ is a trace on $\mathscr{A}$, then $\varphi(z)=\varphi\left(u z u^{*}\right)$ and so by (3. 20), $z=u z u^{*}$, and hence $z \in \mathscr{Z}(\mathscr{A})$.

We now prove necessity of conditions (3.21) and (3.22). If $\delta$ extends $\delta_{0}$, then for $x \in \mathscr{D}_{0}, \gamma \in \Gamma$ :

$$
\begin{aligned}
u(\gamma) \delta_{0}(u(\gamma) * x u(\gamma)) u(\gamma) *-\delta_{0}(x) & \\
= & u(\gamma)\left[\delta(u(\gamma) *) x u(\gamma)+u(\gamma) * \delta_{0}(x) u(\gamma)+u(\gamma) * x \delta(u(\gamma))\right] u(\gamma) * \\
& -\delta_{0}(x) \\
= & {[-\delta(u(\gamma)) u(\gamma) *, x] }
\end{aligned}
$$

noting that by (2.2)

$$
\delta(u(\gamma) *)=-u(\gamma) * \delta(u(\gamma)) u(\gamma) *
$$

and that $\delta(u(\gamma)) u(\gamma) * \in \mathscr{A}^{\tau}$ if $\delta$ commutes with $\tau$. If $\varphi$ is any trace on $\mathscr{A}$, then by (2.1), $u \rightarrow \varphi\left[\delta(u) u^{*}\right]$ is a homomorphism on the unitary part of $\mathscr{D}$. Hence (3.22) holds. Conversely, suppose (3.21) and (3.22) hold. Define $\Phi$ by 3. 7. Then $u\left(\gamma_{1}\right) \Phi\left(\gamma_{2}, \gamma_{3}\right) u\left(\gamma_{1}\right) *=\Phi\left(\gamma_{2}, \gamma_{3}\right)$, $\gamma_{i} \in \Gamma$, because $\Phi\left(\gamma_{2}, \gamma_{3}\right) \in \mathscr{Z}\left(\mathscr{A}^{\tau}\right) \subset \mathscr{Z}(\mathscr{A})$ by Lemma 3.3 and the above remark. Let $\varphi$ be any trace on $\mathscr{A}$. Then

$$
\begin{aligned}
\varphi\left(\Phi\left(\gamma_{1}, \gamma_{2}\right)\right)= & \varphi\left(b\left(\gamma_{1}+\gamma_{2}\right)\right)-\varphi\left(b\left(\gamma_{1}\right)\right)-\varphi\left[u\left(\gamma_{1}\right) b\left(\gamma_{2}\right) u\left(\gamma_{1}\right) *\right] \\
& -i \varphi\left[u\left(\gamma_{1}+\gamma_{2}\right) \delta_{0}\left(u\left(\gamma_{1}+\gamma_{2}\right) * u\left(\gamma_{1}\right) u\left(\gamma_{2}\right)\right) u\left(\gamma_{2}\right) * u\left(\gamma_{1}\right) *\right] \\
= & \varphi\left(b\left(\gamma_{1}+\gamma_{2}\right)\right)-\varphi\left(b\left(\gamma_{1}\right)\right)-\varphi\left(b\left(\gamma_{2}\right)\right)
\end{aligned}
$$




$$
\begin{aligned}
& -i \varphi\left\{\delta_{0}\left[u\left(\gamma_{1}+\gamma_{2}\right) * u\left(\gamma_{2}\right) u\left(\gamma_{1}\right) u\left(\gamma_{1}\right) * u\left(\gamma_{2}\right) * u\left(\gamma_{1}\right) u\left(\gamma_{2}\right)\right]\right. \\
& \left.\cdot u\left(\gamma_{2}\right) * u\left(\gamma_{1}\right) * u\left(\gamma_{2}\right) u\left(\gamma_{1}\right) u\left(\gamma_{1}\right) * u\left(\gamma_{2}\right) * u\left(\gamma_{1}+\gamma_{2}\right)\right\} \\
= & \varphi\left(b\left(\gamma_{1}+\gamma_{2}\right)\right)-\varphi\left(b\left(\gamma_{1}\right)\right)-\varphi\left(b\left(\gamma_{2}\right)\right) \\
& -i \varphi\left[\delta_{0}\left(u\left(\gamma_{1}+\gamma_{2}\right) * u\left(\gamma_{2}\right) u\left(\gamma_{1}\right)\right) u\left(\gamma_{1}\right) * u\left(\gamma_{2}\right) * u\left(\gamma_{1}+\gamma_{2}\right)\right] \\
& -i \varphi\left[\delta_{0}\left(u\left(\gamma_{1}\right) * u\left(\gamma_{2}\right) * u\left(\gamma_{1}\right) u\left(\gamma_{2}\right)\right) u\left(\gamma_{2}\right) * u\left(\gamma_{1}\right) * u\left(\gamma_{2}\right) u\left(\gamma_{1}\right)\right] \\
= & \varphi\left(b\left(\gamma_{1}+\gamma_{2}\right)\right)-\varphi\left(b\left(\gamma_{1}\right)\right)-\varphi\left(b\left(\gamma_{2}\right)\right) \\
& -i \varphi\left[\delta_{0}\left(u\left(\gamma_{1}+\gamma_{2}\right) * u\left(\gamma_{2}\right) u\left(\gamma_{1}\right)\right) u\left(\gamma_{1}\right) * u\left(\gamma_{2}\right) * u\left(\gamma_{1}+\gamma_{2}\right)\right] \\
= & \varphi\left(\Phi\left(\gamma_{2}, \gamma_{1}\right)\right)
\end{aligned}
$$

where we have used (2.1) and (3.22). But $\Phi\left(\gamma_{1}, \gamma_{2}\right) \in \mathscr{Z}\left(\mathscr{A}^{\tau}\right)_{h}$ by Lemma 3. 3. Hence $\Phi\left(\gamma_{1}, \gamma_{2}\right)=\Phi\left(\gamma_{2}, \gamma_{1}\right)$ by (3.21). Then by Lemma 3.4 and Lemma 3.6 with $\mathscr{D}=\mathscr{Z}\left(\mathscr{A}^{\tau}\right)_{h}$, there exists a map $z: \Gamma \rightarrow$ $\mathscr{Z}\left(\mathscr{A}^{\tau}\right)$ such that $z(0)=0$ and $\Phi\left(\gamma_{1}, \gamma_{2}\right)=z\left(\gamma_{1}+\gamma_{2}\right)-z\left(\gamma_{1}\right)-z\left(\gamma_{2}\right)$. Thus by Lemma $3.5, \delta$ extends to a derivation on $\mathscr{D}$ commuting with $\tau$. The final remark is clear by uniqueness of Fourier decompositions.

Remark 3.9. The hypotheses of Theorem 3.8 eliminate the "twist" from $\Phi$. Here we explain why this is necessary. The existence of the family of unitaries $\{u(\gamma): \gamma \in \Gamma\}$ leads to an action $\eta: \Gamma \longmapsto$ Aut $\left(\mathscr{Z}\left(\mathscr{A}^{\tau}\right)\right)$ where

$$
\eta(\gamma)(b)=u(\gamma) b u(\gamma)^{*}, b \in \mathscr{Z}\left(\mathscr{A}^{\tau}\right), \gamma \in \Gamma \text {. }
$$

The $u$-twisted 2-cocycle $\Phi$ defines a group

$$
\Gamma_{\phi}=\left\{(\gamma, b): \gamma \in \Gamma, b \in \mathscr{Z}\left(\mathscr{A}^{\tau}\right)_{h}\right\}
$$

by

$$
(\gamma, b)+\left(\gamma^{\prime}, b^{\prime}\right)=\left(\gamma+\gamma^{\prime}, \Phi\left(\gamma, \gamma^{\prime}\right)+\eta(\gamma)\left(b^{\prime}\right)+b\right)
$$

and hence $\Phi$ determines an element $\zeta_{\Phi}$ of the second cohomology group $H_{\eta}^{2}\left(\Gamma, \mathscr{Z}\left(\mathscr{A}^{\tau}\right)_{h}\right)$ where we use the notation of [11]. Then (3.9) is equivalent to $\zeta_{\Phi}$ being zero. Thus a necessary and sufficient condition for (3.9) to hold is that the exact sequence

$$
0 \rightarrow \mathscr{Z}\left(\mathscr{A}^{\tau}\right)_{h} \longrightarrow \Gamma_{\Phi} \longrightarrow \Gamma \longrightarrow 0
$$

split (see [11] again). Unfortunately simple criteria for $\Phi$, as defined by (3.7), to define a sequence (3.23) which splits do not appear to exist except when $\eta$ is trivial where we have the necessary and sufficient condition that $\Phi$ be symmetric.

Note that $H^{2}\left(\Gamma, \mathscr{Z}\left(\mathscr{A}^{\tau}\right)_{h}\right)=(0)$ if $\Gamma=\boldsymbol{Z}$. 


\section{§4. An Example}

Let $G=\boldsymbol{T}^{2}, \quad \Gamma=\boldsymbol{Z}^{2}$. For $\gamma_{1}=\left(m_{1}, n_{1}\right), \gamma_{2}=\left(m_{2}, n_{2}\right) \in \Gamma$, define $\left[\gamma_{1}, \gamma_{2}\right]=m_{1} n_{2}-m_{2} n_{1}$, and $\omega\left(\gamma_{1}, \gamma_{2}\right) \in C[0,1]$ by $\omega\left(\gamma_{1}, \gamma_{2}\right)(t)=\exp \{i t$ $\left.\left[\gamma_{1}, \gamma_{2}\right] / 2\right\}, t \in[0,1]$. Then $\overline{\operatorname{lin}}\left\{\omega\left(\gamma_{1}, \gamma_{2}\right): \gamma_{i} \in \Gamma\right\}=C[0,1]$, and

$$
\omega\left(\gamma_{1}, \gamma_{2}\right) \omega\left(\gamma_{1}+\gamma_{2}, \gamma_{3}\right)=\omega\left(\gamma_{1}, \gamma_{2}+\gamma_{3}\right) \omega\left(\gamma_{2}, \gamma_{3}\right)
$$

for $\gamma_{1}, \gamma_{2}, \gamma_{3}$ in $\Gamma$. Let $K$ denote the Hilbert space $l^{2}\left(\Gamma, L^{2}[0,1]\right)$, and define unitaries $\{W(\gamma): \gamma \in \Gamma\}$ on $K$ by

$$
\left(W\left(\gamma_{2}\right) f\right)\left(\gamma_{1}\right)=\omega\left(\gamma_{1}, \gamma_{2}\right) f\left(\gamma_{1}+\gamma_{2}\right)
$$

for $f \in K, \gamma_{1}, \gamma_{2} \in \Gamma$, and where we let $C[0,1]$ act on $L^{2}[0,1]$ by pointwise multiplication. Then by (4.1)

$$
W\left(\gamma_{1}\right) W\left(\gamma_{2}\right)=\omega\left(\gamma_{1}, \gamma_{2}\right) W\left(\gamma_{1}+\gamma_{2}\right)
$$

for $\gamma_{1}, \gamma_{2} \in \Gamma$, and where we let $C[0,1]$ act on $K$ through its action on $L^{2}[0,1]$ alone.

Let $\mathscr{A}$ denote the $C^{*}$-algebra generated by $\{W(\gamma): \gamma \in \Gamma\}$. Then by (4.2), $\mathscr{A} \supset C[0,1]$, and

$$
\mathscr{A}=\varlimsup\{C[0,1] W(\gamma): \gamma \in \Gamma\} .
$$

Define a strongly continuous unitary representation $U$ of $G$ on $K$ by

$$
\left(U_{g} f\right)(\gamma)=\langle\gamma, g\rangle^{-1} f(\gamma)
$$

$f \in K, g \in G, \gamma \in \Gamma$. Then

$$
U_{g} W(\gamma) U_{g}^{*}=\langle\gamma, g\rangle W(\gamma)
$$

for $g \in G, \gamma \in \Gamma$. Hence

$$
\tau_{g}=\left.A d\left(U_{g}\right)\right|_{A}
$$

defines a strongly continuous action of $G$ on $\mathscr{A}$ such that $W(\gamma) \in \mathscr{A}^{\tau}(\gamma)$ for each $\gamma$ in $\Gamma$. It is clear from (4.2) that $C[0,1] \subseteq \mathscr{A}^{\tau}$, and in fact it is easy to see using $P=\int_{G} \tau(g) d g$ that $\mathscr{A}^{\tau}=C[0,1]$.

Let $\mathscr{D}_{0}=C^{1}[0,1]$, and $\delta_{0}$ denote differentiation on $\mathscr{D}_{0}$. Then $W$ satisfies conditions (3.1-3.3). In fact, note that $W(\gamma)$ commutes with $C[0,1]$ so that in this case

$$
C[0,1]=\mathscr{A}^{\tau}=\mathscr{Z}\left(\mathscr{A}^{\tau}\right)=\mathscr{Z}(\mathscr{A}) \text {. }
$$

If $\varphi$ is any state on $\mathscr{A}^{\tau}, \varphi \circ P$ is a trace on $\mathscr{A}$, so that (3.22) holds. 


\section{Moreover}

$$
W(\gamma) \delta_{0}(W(\gamma) *(\cdot) W(\gamma)) W(\gamma) *-\delta_{0}(\cdot) \equiv 0
$$

so that one can take $b(\gamma) \equiv 0$. However, if $\gamma_{1}, \gamma_{2} \in \Gamma$, and

$$
W=W\left(\gamma_{1}\right) * W\left(\gamma_{2}\right) * W\left(\gamma_{1}\right) W\left(\gamma_{2}\right)=\overline{\omega\left(\gamma_{2}, \gamma_{1}\right)} \omega\left(\gamma_{1}, \gamma_{2}\right)
$$

then $W$ is the function $t \in[0,1] \rightarrow \exp \left(i t\left[\gamma_{1}, \gamma_{2}\right]\right)$, so that $\delta_{0}\left(W^{*}\right) W=$ $i\left[\gamma_{1}, \gamma_{2}\right]$ and (3.22) cannot possibly hold.

\section{Acknowledgements}

The first and third authors are grateful for the financial support of visiting fellowships at the Institute for Advanced Studies, Australian National University during the period of this work.

\section{References}

[ 1] Bratteli, O., Elliott, G. A. and Jørgensen, P.E. T., Decomposition of unbounded derivations into invariant and approximately inner parts, J. Reine Angew. Math., 346 (1984), 166-193.

[2] Bratteli, O. and Evans, D. E., Dynamical semigroups commuting with compact abelian actions, Ergod. Th. and Dynamic Sys., 3 (1983), 187-217.

[3] Bratteli, O. and Jørgensen, P.E.T., Unbounded derivations tangential to compact groups of automorphisms, J. Func. Anal., 48 (1982), 107-133.

[4] Derivations commuting with abelian gauge actions on lattice systems, Commun. Math. Phys, 87 (1982), 353-364.

[5] Goodman, F. and Jørgensen, P. E. T., Unbounded derivations commuting with compact group actions, Commun. Math. Phys, 82 (1981), 399-405.

[6] Goodman, F. and Wassermann, A., Unbounded derivations commuting with compact group actions II, J. Func. Anal., to appear.

[7] Hewitt, E. and Ross, K. A., Abstract Harmonic Analysis I, Springer, Berlin, 1963.

[8] Ikunushi A., Derivations in $C^{*}$-algebras commuting with compact actions, Publ. RIMS Kyoto Univ., 19 (1983), 99-106.

[9] Jørgensen, P.E.T., Compact symmetry groups and generators for sub-markovian semigroups, Z. Wahrsch. Verw. Geb., to appear.

[10] Kishimoto, A. and Robinson, D. W., On unbounded derivations commuting with a compact group of *-automorphisms, Publ. RIMS Kyoto Univ. 18 (1982), 1121-1136.

[11] Maclane, S., Homology, Springer, Berlin, 1963.

[12] Peligrad, C., Derivations of $C^{*}$-algebras which are invariant under an automorphism group I, OT series, 2, Birkhäuser Verlag, 1981, II, Bucuresti preprint 107, 1981.

[13] Pusz, W. and Woronowicz, S. L., Passive states and KMS states for general quantum systems, Commun. Math. Phys., 58 (1978), 273-290. 